\title{
Gut microbiota regulates mouse behaviors through glucocorticoid receptor pathway genes in the hippocampus
}

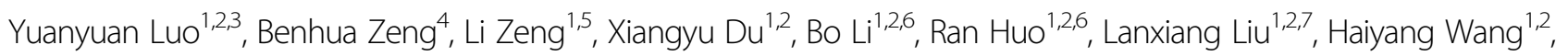
Meixue Dong ${ }^{1,2,7}$, Junxi Pan ${ }^{1,2,6}$, Peng Zheng ${ }^{1,2,7}$, Chanjuan Zhou ${ }^{1,2}$, Hong Wei ${ }^{2}$ and Peng Xie ${ }^{2,3,6,7,8}$

\begin{abstract}
Gut microbiota has an important role in the immune system, metabolism, and digestion, and has a significant effect on the nervous system. Recent studies have revealed that abnormal gut microbiota induces abnormal behaviors, which may be associated with the hypothalamic-pituitary-adrenal (HPA) axis. Therefore, we investigated the behavioral changes in germ-free (GF) mice by behavioral tests, quantified the basal serum cortisol levels, and examined glucocorticoid receptor pathway genes in hippocampus using microarray analysis followed by real-time PCR validation, to explore the molecular mechanisms by which the gut microbiota influences the host's behaviors and brain function. Moreover, we quantified the basal serum cortisol levels and validated the differential genes in an Escherichia coli-derived lipopolysaccharide (LPS) treatment mouse model and fecal "depression microbiota" transplantation mouse model by real-time PCR. We found that GF mice showed antianxiety- and antidepressant-like behaviors, whereas E. coli LPS-treated mice showed antidepressant-like behavior, but did not show antianxiety-like behavior. However, "depression microbiota" recipient mice exhibited anxiety- and depressive-like behaviors. In addition, six glucocorticoid receptor pathway genes (SIc22a5, Aqp1, Stat5a, Ampd3, Plekhf1, and Cyb561) were upregulated in GF mice, and of these only two (Stat5a and Ampd3) were upregulated in LPS-treated mice, whereas the shared gene, Stat5a, was downregulated in "depression microbiota" recipient mice. Furthermore, basal serum cortisol levels were decreased in E. coli LPS-treated mice but not in GF mice and "depression microbiota" recipient mice. These results indicated that the gut microbiota may lead to behavioral abnormalities in mice through the downstream pathway of the glucocorticoid receptor. Herein, we proposed a new insight into the molecular mechanisms by which gut microbiota influence depressive-like behavior.
\end{abstract}

\section{Introduction}

The human gastrointestinal tract contains approximately trillion microorganisms, most of which are bacteria, belonging to $>1000$ bacterial species $^{1}$. Bacteroidetes and Firmicutes constitute over $90 \%$ of the species in the gut microbiota ${ }^{2}$. Preclinical and clinical evidence have

Correspondence: Hong Wei (weihong63528@163.com) or Peng Xie (xiepeng@cqmu.edu.cn)

${ }^{1}$ Institute of Neuroscience and the Collaborative Innovation Center for Brain Science, Chongqing Medical University, Chongqing 400016, China

${ }^{2}$ Chongqing Key Laboratory of Neurobiology, Chongqing 400016, China

Full list of author information is available at the end of the article.

These authors are contributed equally: Yuanyuan Luo, Benhua Zeng, Li Zeng, Xiangyu Du indicated that the gut microbiota plays an important role in a wide range of host processes in both healthy population and patients ${ }^{3,4}$. In particular, recent studies have hinted that the gut microbiota can have dramatic effects on the development and function of the host brain ${ }^{5}$.

Recently, accumulating studies have revealed bidirectional communication between the intestinal microbiota and the brain, and proposed a novel conceptual model of a "microbiota-gut-brain axis"6-8. The gut-brain axis is an interaction system that integrates immunological,

\section{(c) The Author(s) 2018}

(c) (i) Open Access This article is licensed under a Creative Commons Attribution 4.0 International License, which permits use, sharing, adaptation, distribution and reproduction in any medium or format, as long as you give appropriate credit to the original author(s) and the source, provide a link to the Creative Commons license, and indicate if changes were made. The images or other third party material in this article are included in the article's Creative Commons license, unless indicated otherwise in a credit line to the material. If material is not included in the article's Creative Commons license and your intended use is not permitted by statutory regulation or exceeds the permitted use, you will need to obtain permission directly from the copyright holder. To view a copy of this license, visit http://creativecommons.org/licenses/by/4.0/. 
neural, endocrine and metabolic pathways, and dysfunction of this axis has pathophysiological effects on the host $^{9,10}$. Many studies have found a high prevalence of mood disorders such as depression and/or anxiety in patients with gastrointestinal disorders including irritable bowel disorder and inflammatory bowel disorder ${ }^{11,12}$, which indicated the importance of the gut-brain axis in the pathophysiology of mood disorders. The gut and the brain may communicate through multiple mechanisms, including immune responses, vagus nerve, short-chain fatty acids, endocrine signaling and tryptophan metabo$\operatorname{lism}^{5,13}$. Our previous studies have revealed that the gut microbiota can modulate anxiety- and depressive-like behaviors in mice ${ }^{14-16}$, and lead to molecular changes in the hippocampus ${ }^{17,18}$, hypothalamus ${ }^{15}$, and liver ${ }^{16}$. However, the molecular mechanisms of the communication between gut microbiota and brain are not fully understood.

Germ-free (GF) mice, born and raised without exposure to microbes, is a powerful tool for understanding the effects of the gut microbiota on the host. Previous studies have demonstrated that GF mice had higher levels of serum adrenocorticotrophic hormone (ACTH) and corticosterone following acute stress compared with specific pathogen-free (SPF) mice ${ }^{19,20}$. Moreover, GF mice exhibited antianxiety-like behavior and increased plasma corticosterone levels compared with SPF mice, and the elevated plasma corticosterone may also be owing to the stress of acclimatization ${ }^{21}$. GF rats showed increased peripheral corticosterone levels after stress and a decreased glucocorticoid receptor $(\mathrm{Nr} 3 \mathrm{c} 1)$ mRNA level in the hippocampus ${ }^{22}$. In addition, plasma $\mathrm{ACTH}$ and corticosterone levels were decreased in Bifidobacterium infantis-monoassociated mice, whereas they were increased in Escherichia coli-monoassociated mice ${ }^{20,23}$, suggesting the important role of corticosterone in gut microbiota alterations. Therefore, we speculated that the disturbances in glucocorticoid receptor pathway are the important mechanisms by which the gut microbiota influences the host's behaviors and brain function.

To test this hypothesis, we investigated the behavioral changes in GF mice, the basal serum cortisol levels and the alterations in expression of glucocorticoid receptor pathway genes in hippocampus. More importantly, we compared the present results with those of the E. coliderived lipopolysaccharide (LPS) treatment mouse model and the fecal "depression microbiota" transplantation (FMT) mouse model. Finally, we further analyzed the expression alterations of the glucocorticoid receptor pathway genes in hippocampus accompanied with behavioral changes, to explore the potential mechanisms by which the gut microbiota influences the host's behaviors.

\section{Material and methods}

\section{Animals}

Eight-week-old male GF $(n=19)$ and SPF $(n=20)$ $\mathrm{BALB} / \mathrm{c}$ mice were provided by the animal experimental center of the Third Military Medical University (Chongqing, China). GF mice were fed in flexible film plastic isolators. All conditions were kept sterile and verified to meet the Chinese Laboratory Animal Microbiological Standards and Monitoring (GB 14922.2-2011) by testing the feces and skin of the GF mice. GF and SPF mice were housed five per cage under a 12-h light/dark cycle (lights on at 07:30-19:30), a constant temperature of $21-22^{\circ} \mathrm{C}$ and a relative humidity of $55 \pm 5 \%$. Autoclaved standard mice chow of the same formulation and water ad libitum was given to all animals. This study was approved by the Ethics Committee of Chongqing Medical University and conducted in accordance with the National Institutes of Health Guide for the Care and Use of Laboratory Animals (NIH Publication No. 80-23).

\section{E. coli LPS-treated mice}

Thirteen 6-week-old male SPF mice were given daily drinking water containing E. coli LPS (Sigma-Aldrich Ltd., St. Louis, MO, USA) at a concentration of $20 \mu \mathrm{g} / \mathrm{ml}$ for 2 weeks, and the consumption of drinking water was not limited. The control SPF mice (male, 6 weeks old, $n=13$ ) were given daily drinking water without $E$. coli LPS. All other conditions between the two groups were consistent. Behavioral tests were performed two weeks later, followed by tissue sample collection.

\section{FMT mice}

As previously described ${ }^{14}$, the FMT mouse model $(n=$ 30) was established by transferring fecal samples of severe depressive patients to GF mice, and the control mice $(n=$ 30) were transplanted with normal human feces. The two groups were called "depression microbiota" recipient mice group and "healthy microbiota" recipient mice group, respectively. Behavioral tests were performed, and tissue samples were collected after 2 weeks. In addition, the timelines of above-mentioned mouse models were displayed in Supplementary method. S1.

\section{Open field test (OFT)}

The GF and SPF mice were moved to the testing room for acclimation at least $1 \mathrm{~h}$ prior to behavioral testing. Mice were placed individually in the middle of the open field apparatus $(45 \times 45 \times 45 \mathrm{~cm})$ and allowed to adapt for $1 \mathrm{~min}$, then the spontaneous activities were recorded for 5 min using the SMART 2.5 software (Panlab, Barcelona, Spain). The total movement distance and the percentage of center distance (inner $25 \%$ of the surface area) were used as indexes of locomotor activity and anxiety-like 
behavior, respectively. After each mouse was tested, the odor was eliminated with $70 \%$ alcohol.

\section{Forced swim test (FST)}

Mice were placed in a Plexiglas cylinder $(30 \mathrm{~cm}$ high, $15 \mathrm{~cm}$ diameter) filled with water $\left(25 \pm 1^{\circ} \mathrm{C}\right)$ to $18 \mathrm{~cm}$. The test lasted $6 \mathrm{~min}$, and in the last $5 \mathrm{~min}$ we recorded the immobility/absence of all motion with the exception of minimal movements to keep the head above the water. Immobility time was used to evaluate the depressive-like behavior of the mice. The water was completely replaced after each test.

\section{Novelty suppressed feeding test (NSFT)}

All mice were food-deprived for $24 \mathrm{~h}$ prior to the test. Each mouse was placed in the corner of the open field apparatus $(45 \times 45 \times 45 \mathrm{~cm})$ with a single food pellet in the center. The latency to feed, an index of depressive-like behavior and motivation level of the mouse, was recorded. Then, the mouse was immediately returned to its home cage and the food consumption during the subsequent 5 min was measured to eliminate the effect of appetite on the latency time.

\section{Sample collection and preparation}

Mice were killed in a random order by cervical dislocation after being anesthetized with $10 \%$ chloral hydrate $(400 \mathrm{mg} / \mathrm{kg})^{15}$. The brain was quickly removed from the cranium, and hippocampus were dissected out on ice-cold plate and frozen in liquid nitrogen, then stored at $-80{ }^{\circ} \mathrm{C}$ before assay. Blood samples were collected in a plastic tube. The serum was obtained by centrifugation $(3000 \mathrm{~g}$, $10 \mathrm{~min}, 4^{\circ} \mathrm{C}$ ) and stored at $-80^{\circ} \mathrm{C}$ for subsequent analysis. To avoid fluctuations in the results owing to the circadian rhythm of hypothalamic-pituitary-adrenal (HPA) axis, samples in each group were collected at the same time of day (between 9:00 and 11:00 h) ${ }^{20}$.

\section{Microarray analysis}

The Glucocorticoid Signaling PCR Microarrays (SABiosciences Corporation, Frederick, MD, USA) were used to analyze the alterations in glucocorticoid receptor pathway genes. In detail, three arrays were used for the GF mice and three for the SPF mice. Three mouse hippocampus were mixed into a pooled RNA sample, and all experiments in GF mice group and SPF mice group were performed in triplicate using distinct RNA samples. Total RNA from each sample were extracted with TRIzol extraction procedures (Invitrogen, Carlsbad, CA, USA). Then, the total RNA from each sample were purified by RNeasy $^{\circledast}$ MinElute $^{\text {rw }}$ Cleanup Kit (Invitrogen) and the RNA concentration and purity were tested by NanoDrop ND-1000 spectrophotometer (Thermo Fisher Scientific, Waltham, MA, USA). The RNA samples were examined for the 84 related genes in the glucocorticoid receptor pathway (Supplementary Table. 1). Data were analyzed with the $2^{-\Delta \Delta C t}$ method and exhibited in the form of fold change. Student's $t$ test was used to select the different genes between the two groups $(p<0.05)$. The $q$ value method was employed to control the false discovery rate (set to 0.1 ) associated with multiple analysis ${ }^{24,25}$.

\section{Bioinformatics analysis}

Ingenuity Pathway Analysis (IPA) software (Qiagen, Shanghai, China) was used to analyze the biological functions and relevant networks of differentially expressed genes, and the enrichment of genes and diseases/ functions.

\section{Quantitative real-time PCR (qRT-PCR)}

qRT-PCR was conducted as previously described ${ }^{18}$. In brief, RNA was extracted from hippocampal tissues of mice according to the TRIzol extraction protocol (Thermo Fisher Scientific, MA, USA), and reversetranscribed into cDNA with the PrimeScript ${ }^{\mathrm{mm}}$ RT reagent Kit (Takara, Dalian, China). Subsequently, qRTPCR was performed with the LightCycler 96 system (Roche, Mannheim, Germany) with GoTaq ${ }^{\circledast}$ 1-Step RTqPCR System for Dye-Based Detection (Promega, Madison, WI, USA). The primers were obtained from Sangon Biotech (Shanghai, China). Geometric mean of Gapdh and $A c t b$ mRNA was used to normalize the target mRNA $^{26}$.

\section{Serum cortisol measurement}

The basal serum cortisol levels of three mice models were quantified using a Mouse Cortisol Enzyme-linked Immunosorbent Assay (ELISA) Kit (Sangon Biotech, Shanghai, China) as recommended by the manufacturer's protocol. Optical absorbance at $450 \mathrm{~nm}$ was measured with a microplate reader (Bio-Rad Co., California, USA). A standard curve was constructed $(15-240 \mathrm{ng} / \mathrm{ml})$ and the concentrations of cortisol in serum samples were determined from the standard curve.

\section{Statistical analysis}

The results were analyzed using nonparametric tests or Student's $t$ test, where appropriate. All statistical analyzes were performed using SPSS 20.0 software (IBM, Armonk, $\mathrm{NY}$, USA). Data are presented as mean \pm SEM. A value of $p<0.05$ was considered statistically significant.

\section{Results}

GF mice exhibit antianxiety- and antidepressant-like behaviors

As previously described, we explored the effects of gut microbiota on mice behaviors by comparing GF and SPF mice. In the OFT, the GF mice showed a significantly 


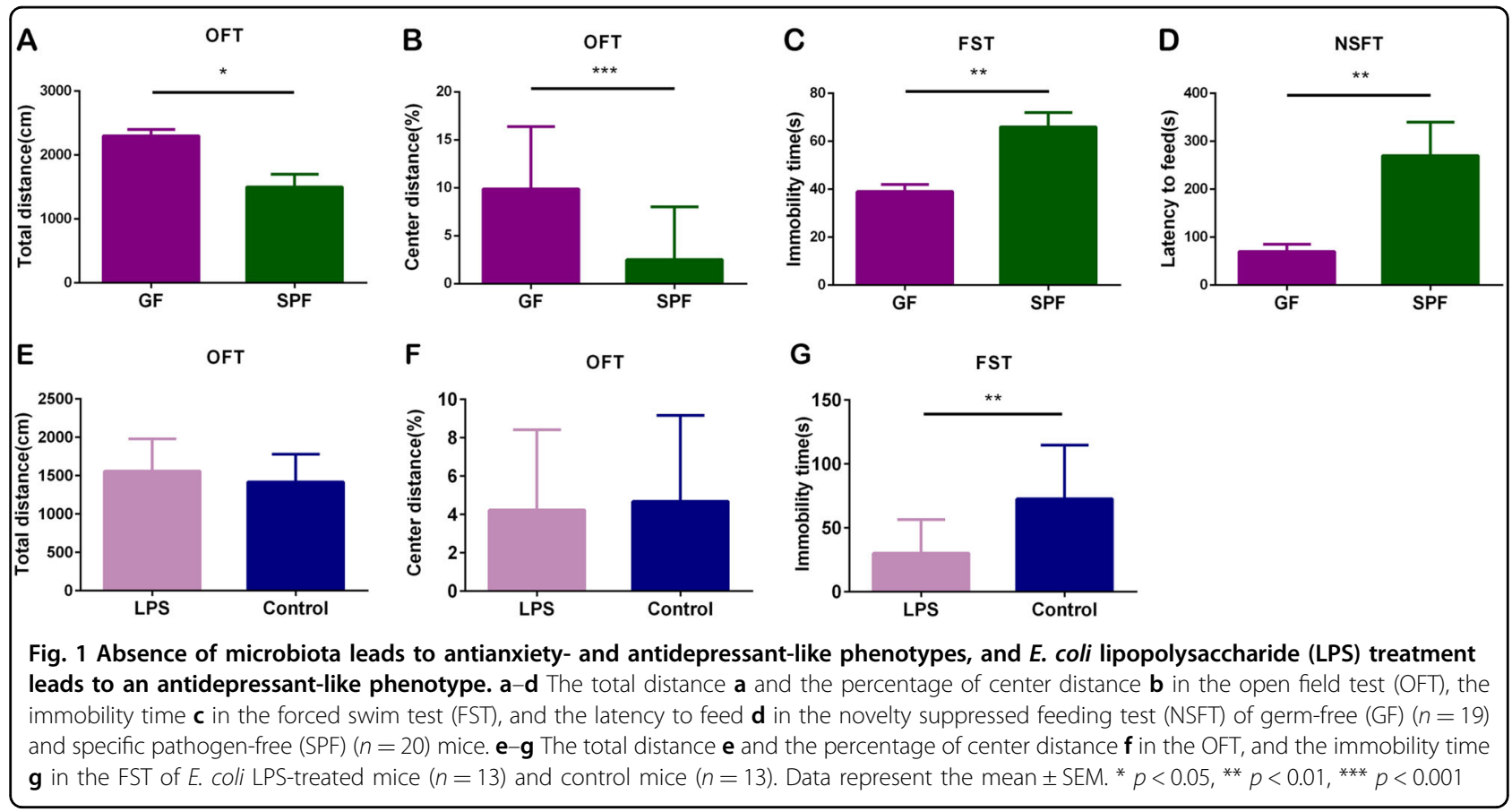

increased total distance (Fig. 1a, $p<0.05$ ) and increased percentage of center distance (Fig. 1b, $p<0.001$ ) compared with the SPF mice, indicating higher locomotor activity and antianxiety-like behavior in GF mice. Moreover, the GF mice exhibited less immobility time than the SPF mice in the FST (Fig. 1c, $p<0.01$ ) and showed decreased latency to feed in the NSFT (Fig. 1d, $p<0.01$ ), suggesting antidepressant-like behavior in GF mice.

E. coli LPS-treated mice show antidepressant-like behavior In current study, E. coli LPS-treated mice showed less immobility time than the control mice in the FST (Fig. 1g, $p<0.01)$. However, in OFT, there were no differences in the total distance (Fig. 1e) or the percentage of center distance (Fig. 1f) between E. coli LPS-treated mice and control mice. These results suggest that the E. coli LPStreated mice exhibit antidepressant-like behavior, but not antianxiety-like behavior.

\section{"Depression microbiota" recipient mice exhibit anxiety- and depressive-like behaviors}

Previously we have reported the "depression microbiota" recipient mice exhibited anxiety- and depressivelike behaviors compared with "healthy microbiota" recipient mice ${ }^{14}$. Namely, in OFT, the percentage of center distance of "depression microbiota" recipient mice was decreased compared with "healthy microbiota" recipient mice. Moreover, the immobility time in FST and tail suspension test was increased in "depression microbiota" recipient mice.
Table 1 Differentially expressed genes in the microarray

\begin{tabular}{llllllll}
\hline Gene & FC & $\boldsymbol{p}$ value & FDR & Gene & FC & $\boldsymbol{p}$ value & FDR \\
\hline Slc10a6 & 22.81 & $<0.001$ & $<0.001$ & Pou2f1 & 1.29 & 0.021 & 0.042 \\
Slc22a5 & 1.46 & 0.002 & 0.025 & Bmper & 1.33 & 0.023 & 0.042 \\
Tsc22d3 & 2.30 & 0.004 & 0.025 & Bcl6 & 1.28 & 0.023 & 0.042 \\
Aqp1 & 7.39 & 0.005 & 0.025 & Plekhf1 & 2.06 & 0.028 & 0.043 \\
Mertk & 1.73 & 0.005 & 0.025 & Creb1 & 1.21 & 0.028 & 0.043 \\
Stat5a & 1.55 & 0.006 & 0.028 & Arid5b & 1.33 & 0.029 & 0.043 \\
Ampd3 & 1.40 & 0.008 & 0.029 & Adarb1 & 1.18 & 0.031 & 0.043 \\
Ghrhr & 11.99 & 0.009 & 0.030 & Sgk1 & 1.93 & 0.032 & 0.043 \\
Pld1 & 1.55 & 0.011 & 0.034 & Cyb561 & 1.24 & 0.034 & 0.044 \\
Fkbp5 & 1.32 & 0.013 & 0.036 & Usp2 & 1.17 & 0.044 & 0.054 \\
Aff1 & 1.38 & 0.020 & 0.042 & Glul & 1.18 & 0.049 & 0.058 \\
Per1 & 1.26 & 0.020 & 0.042 & & & & \\
\hline
\end{tabular}

Absence of gut microbiota upregulates the expression of glucocorticoid receptor pathway genes

We examined 84 genes related to the glucocorticoid receptor pathway in the hippocampus of GF and SPF mice by using microarray. A total of 23 genes showed significant differences between the two groups ( $p$ value $<$ 0.05 , FDR $<0.1$ ) (Table 1). Among them, Slc10a6 (fold change $(\mathrm{FC})=22.81)$, Ghrhr $(\mathrm{FC}=11.99)$, and Aqp1 (FC $=7.39$ ) had the highest FC values. These results revealed that the absence of gut microbiota significantly 
upregulated the glucocorticoid receptor pathway genes in hippocampus.

\section{Differentially expressed genes in hippocampus are associated with neurological disorders}

We used IPA software to analyze the 23 differentially expressed genes and found that these genes were significantly enriched in neurological disorders (Supplementary Figure S1) and highly related to cell development, cell proliferation, cell death, and cell survival (Supplementary Figure S2).

\section{qRT-PCR validation}

We used PCR to validate the 23 differentially expressed genes in microarray analysis. Six genes, including Slc22a5, Aqp1, Stat5a, Ampd3, Plekhf1 and Cyb561 were upregulated in GF mice compared with SPF mice (Fig. 2a). Moreover, these six significantly expressed genes were further validated in the hippocampus of $E$. coli LPStreated mice and "depression microbiota" recipient mice. Two of them, Stat5a and Ampd3 were upregulated in E. coli LPS-treated mice ( $p$ value $<0.05$, FDR $<0.1)$ (Fig. $2 \mathrm{~b}$ ). However, Stat $5 a \quad(\mathrm{FC}=0.84)$ was downregulated in "depression microbiota" recipient mice compared with "healthy microbiota" recipient mice ( $p$ value $<0.01$, FDR $<0.1$ ) (Fig. 2c). The details of these genes in three mouse models are displayed in Table 2 and Fig. 3.

\section{E. coli LPS-treated mice show decreased basal serum cortisol levels and the other two mouse models show no change}

In present study, E. coli LPS-treated mice showed a decreased basal serum cortisol levels compared with control mice (Fig. $4 \mathrm{~b}, p<0.05$ ). However, the basal serum cortisol levels did not change between GF mice and SPF mice (Fig. 4a), as well as between "depression microbiota" recipient mice and "healthy microbiota" recipient mice (Fig. 4c).

\section{Discussion}

Recently, studies on the effects of gut microbiota on mice behaviors have been highlighted, and absence of microbiota may lead to behavioral abnormalities in mice through the HPA axis ${ }^{20,21}$. In current study, GF mice showed antianxiety- and antidepressant-like behaviors, and the targeted microarray analysis found 23 upregulated glucocorticoid receptor pathway genes in the hippocampus of GF mice. These genes are highly associated with neurological disorders. PCR validation confirmed that six genes (Slc22a5, Aqp1, Stat5a, Ampd3, Plekhf1, and $C y b 561$ ) were upregulated in the hippocampus of GF mice compared with SPF mice. In addition, E. coli LPStreated mice showed antidepressant-like behavior, but did not show antianxiety-like behavior compared with the control mice. Of these six genes, two (Stat5a and Ampd3) were upregulated in $E$. coli-derived LPS-treated mice. Moreover, the "depression microbiota" recipient mice exhibited anxiety- and depressive-like behaviors ${ }^{14}$, and the Stat5a gene was downregulated in their hippocampus compared with the "healthy microbiota" recipient mice. In addition, basal serum cortisol levels reduced in E. coliderived LPS-treated mice model, but did not change in GF mice model and "depression microbiota" recipient mice model.

Consistent with our previous research ${ }^{14,18}$, GF mice exhibited increased percentage of center distance and decreased immobility time compared with SPF mice, suggesting that absence of gut microbiota can lead to antianxiety- and antidepressant-like behaviors. Furthermore, Neufeld et al. have suggested that Swiss Webster female GF mice exhibited antianxiety-like behavior ${ }^{21}$. However, Crumeyrolle-Arias et al. have demonstrated that F344 GF rats displayed anxiety-like behavior compared with SPF rats ${ }^{22}$. We speculated that the different behavioral phenotypes in mice and rats may be related to the genetic background differences ${ }^{27}$.

Plasma ACTH and/or corticosterone levels in GF mice were higher after acute stress than SPF mice, but the basal cortisol levels showed no difference ${ }^{19,20}$. Our current study also showed there was no difference in basal serum cortisol levels between GF mice and SPF mice. However, K. M. Neufeld et al. showed that plasma corticosterone levels in GF mice were increased compared with SPF mice, but the authors also speculated that the elevated plasma corticosterone may be due to the stress of acclimatization ${ }^{21}$. These studies suggested that gut microbiota can affect the reactivity of the HPA axis. Furthermore, abnormal glucocorticoid levels can induce behavioral changes $^{28,29}$. In our research, six glucocorticoid receptor pathway genes (Slc22a5, Aqp1, Stat5a, Ampd3, Plekhf1, and $C y b 561$ ) were upregulated in GF mice, but Stat5a was downregulated in "depression microbiota" recipient mice. Hence, we inferred that Stat5a may be the key mediator in the effects of glucocorticoid on the depressive-like behavior of mice. In addition, both GF mice and E. coli LPStreated mice showed antidepressant-like behavior, and two glucocorticoid receptor pathway-related genes (Stat5a and Ampd3) were upregulated in the two models. We inferred that these two genes may be related to antidepressant-like behavior. Taken together, these findings may provide a further insight into the pathogenesis of depression, and suggest that the Stat5a gene is an important gene in depression.

The janus tyrosine kinase-signal transducer and activator of transcription (STAT) pathway is a pathway by which cells respond to cell growth as well as a variety of extracellular stimuli ${ }^{30,31}$. Stat5a is a member of the STAT family, which mediates intracellular signaling of the 


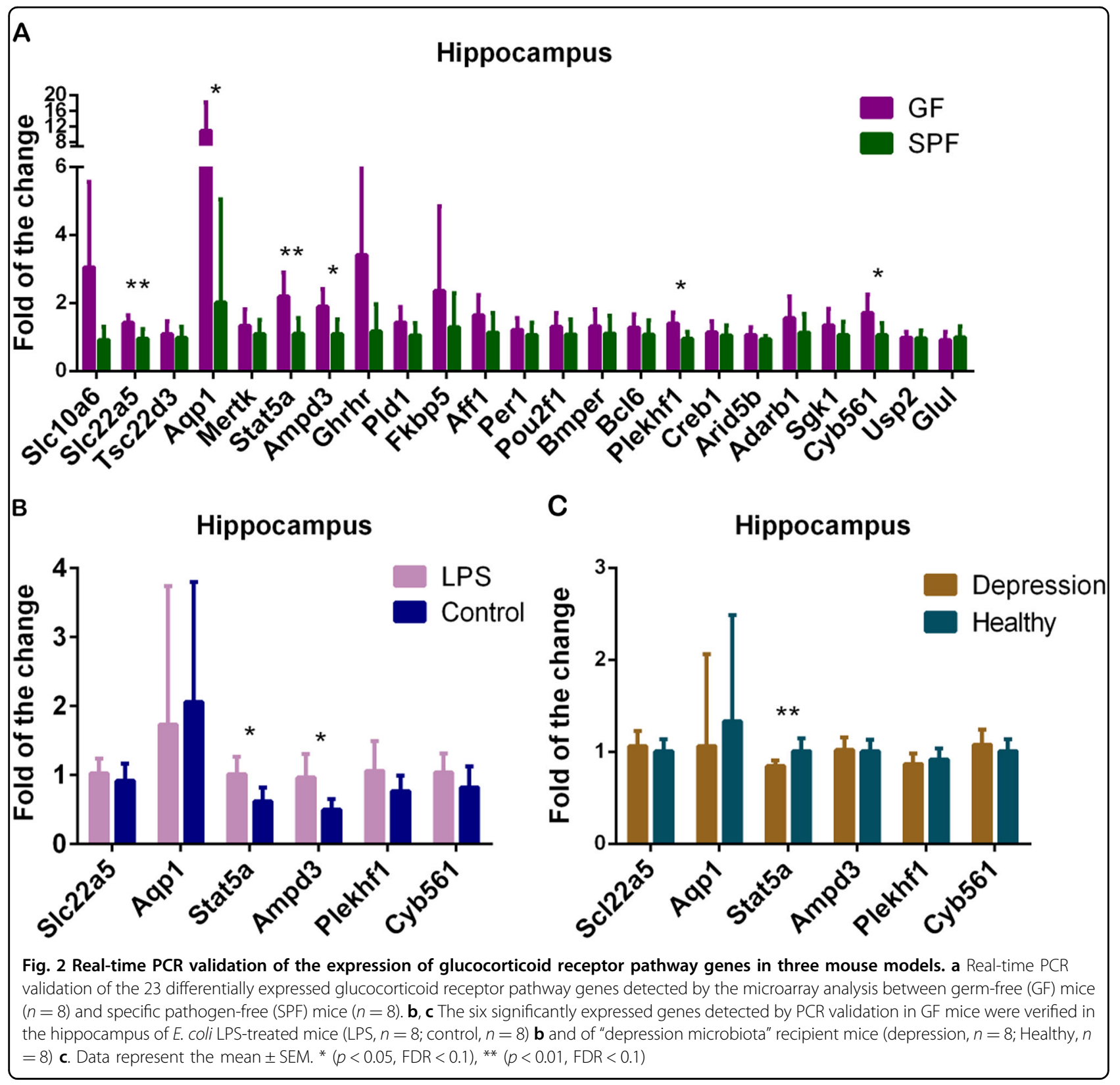

cytokine cell surface receptors, and transmits it to the nucleus ${ }^{31,32}$. It is also essential for cell differentiation, cell proliferation, cell apoptosis ${ }^{32}$, cell cycle regulation, and anti-apoptosis ${ }^{31}$. Sun et al. have found the Stat5a gene was increased in the hippocampus of rats after focal cerebral ischemia and reperfusion, indicating that Stat5a may play a protective role in damaged nerve cells ${ }^{31}$. However, the JAK2-STAT5 signaling pathway mediates interleukin-3-induced activation of microglia, which is associated with the pathogenesis of multiple sclerosis, Alzheimer's disease (AD) and Parkinson's disease ${ }^{33}$. Moreover, locomotor activity, feeding behavior, aggressive and exploratory behaviors may also be associated with cytokine-activated STAT5 signaling ${ }^{34}$. Growth hormone treatment can improve cognitive function ${ }^{35}$, which was accompanied with activation of STAT5 signaling pathway in mouse brain ${ }^{34}$. Hence, we speculated that the Stat5a may be associated with depression, and downregulation can lead to depressive-like behavior.

Aquaporins (AQPs) are the water channels that can transport water across cell membrane in many tissues ${ }^{36}$. Upregulation of Aquaporin 1 (AQP1) caused hippocampal cell edema and delayed cell death following traumatic brain injury ${ }^{37}$, showing that sometimes it protects against neuronal cell damage ${ }^{38}$. However, AQP1 protein overexpression was associated with vacuolization 
in the astroglial cytoplasm in CA1 astrocytes, which may cause cell apoptosis ${ }^{39}$. AQP1 may also lead to cytotoxic edema of the hippocampus by increasing the intracellular $\mathrm{pH}^{40}$. Furthermore, AQP1 has been reported to be

Table 2 Details of the six significant genes identified in the three mouse models

\begin{tabular}{llll}
\hline & GF mice & $\begin{array}{l}\text { LPS treatment } \\
\text { mice }\end{array}$ & $\begin{array}{l}\text { Depression } \\
\text { mice }\end{array}$ \\
& $\begin{array}{l}\text { Antianxiety- and } \\
\text { antidepressant-like } \\
\text { phenotypes }\end{array}$ & $\begin{array}{l}\text { Antidepressant- } \\
\text { like phenotype }\end{array}$ & $\begin{array}{l}\text { Anxiety- and } \\
\text { depressive-like } \\
\text { phenotypes }\end{array}$ \\
\hline Slc22a5 & $\uparrow$ & - & - \\
Aqp1 & $\uparrow$ & - & - \\
Stat5a & $\uparrow$ & $\uparrow$ & $\downarrow$ \\
Ampd3 & $\uparrow$ & $\uparrow$ & - \\
Plekhf1 & $\uparrow$ & - & - \\
Cyb561 & $\uparrow$ & - & - \\
\hline
\end{tabular}

abnormal expression in the brain of AD patients ${ }^{41}$. These studies suggest that the Aqp1 gene may have protective or harmful effects on nerve cells.

Plekhf1 is considered to initiate caspase-independent apoptosis through the lysosomal-mitochondrial pathway $^{42}$. But to our knowledge, there is little study on its role in nervous system. We speculated it may affect the nervous system through cell apoptosis. Cytochrome b561 is considered to be the major transmembrane protein in catecholamines and neuropeptide-secreting vesicles of the pituitary and other neuroendocrine tissues ${ }^{43}$. It can transport electrons across the lipid bilayer to provide transmembrane reduction equivalents, to support dopamine $\beta$-hydroxylase activity and peptidyl $\alpha$-amidating monooxygenase activity ${ }^{44,45}$. Dopamine $\beta$-hydroxylase can hydroxylate dopamine to produce norepinephrine ${ }^{46}$. Dopamine and norepinephrine as neurotransmitters can directly affect behavior.

The Slc22a5 gene encodes an organic cation/carnitine transporter that plays an important role in the pathway of long-chain fatty acids through the internal mitochondrial membrane for $\beta$-oxidation to produce $\mathrm{ATP}^{47}$. Carnitine and/or acetylcarnitine supplementation benefits $\mathrm{AD}$ and

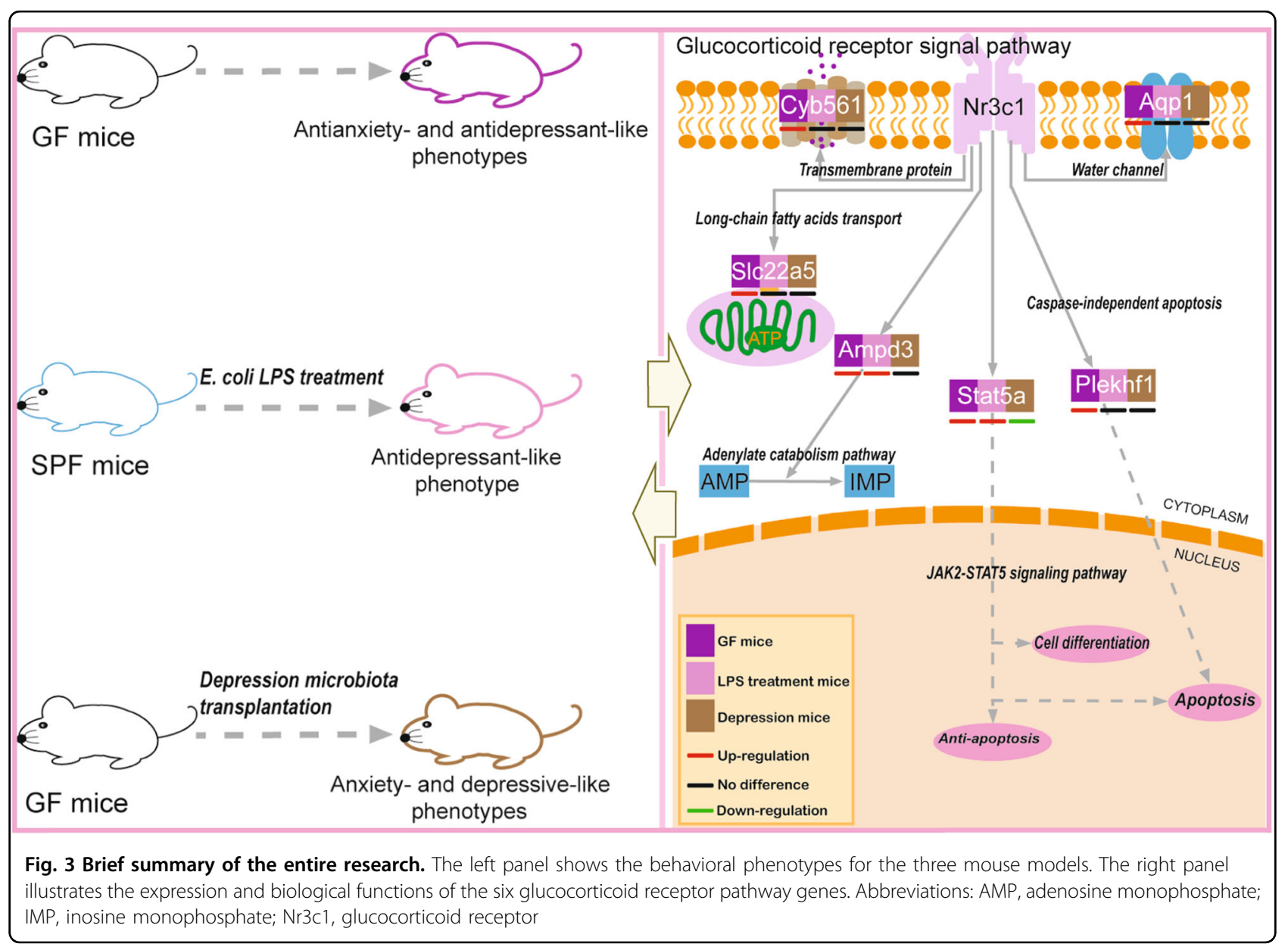




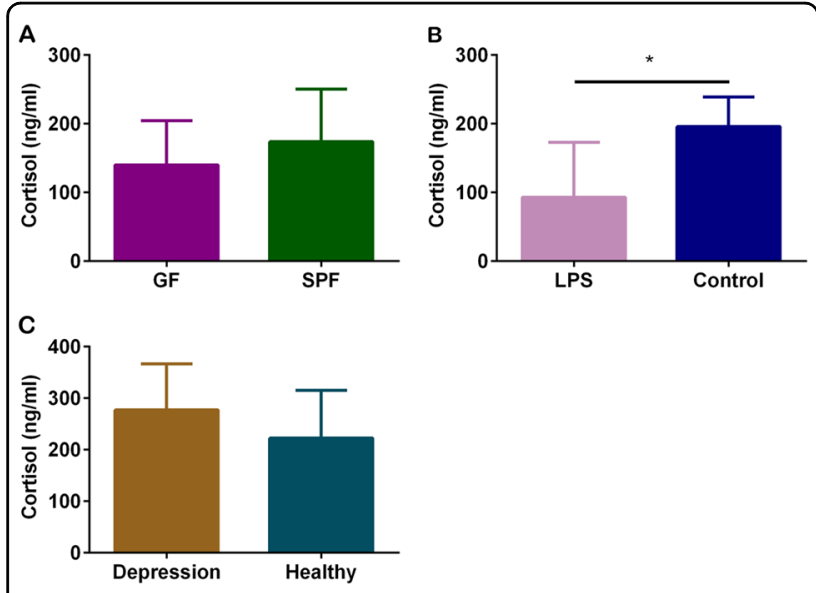

Fig. 4 The basal serum cortisol levels in three mouse models. a The basal serum cortisol levels were quantified between germ-free (GF) mice $(n=6)$ and specific pathogen-free (SPF) mice $(n=6), \mathbf{b}$ between E. coli lipopolysaccharide (LPS)-treated mice $(n=6)$ and control mice $(n=6)$ and $\mathbf{c}$ between "depression microbiota" recipient mice and "healthy microbiota" recipient mice (Depression, $n=6$; Healthy, $n=6$ ). Data represent the mean \pm SEM. ${ }^{*} p<0.05$

depression, as it not only produces ATP by mitochondrial oxidation, but it may enhance mitochondrial function and provide antioxidant effects by providing acetyl moieties ${ }^{48}$. Studies have shown that Slc22a5 expressed in vascular endothelial cells can transport acetylcarnitine through the blood-brain barrier, regulating the concentration of carnitine and acetyl moieties on both sides of the blood-brain barrier ${ }^{47}$. In our research, the elevated Slc22a5 in the hippocampus of GF mice suggests that carnitine transport and/or energy production may be increased, and thus may play a protective role. Furthermore, adenosine monophosphate deaminase 3 (Ampd3) is a key enzyme in the catabolic pathway of adenylate that breaks adenosine monophosphate into inosine monophosphate, and eventually produces uric acid ${ }^{49,50}$. Ampd3 is ubiquitously expressed; it can regulate the energy metabolism of the body and may regulate the energy balance $^{50}$. ATP mediates lipid and protein synthesis, buffers intracellular calcium, and regulates apoptosis and resilience pathways ${ }^{51}$. Energy metabolic disorder is strongly associated with depression ${ }^{52-54}$. Therefore, ATP plays an important role in the brain. In present study, Slc22a5 and Ampd3 were upregulated in GF mice; hence we speculated they may affect brain by modulating ATP levels in hippocampus.

Previous study have shown abnormal gut microbiota can lead to reduced integrity of the intestinal barrier, leading to increased leakage of LPS, which can activate systemic inflammation ${ }^{55}$. Prevention of intestinal barrier impairment and decreasing circulating LPS levels by a probiotic treatment can attenuate the HPA axis response to stress ${ }^{56}$. In current study, oral E. coli LPS treatment induced abnormal behavior and increased glucocorticoid receptor pathway genes in mice. These results were consistent with previous findings that $E$. coli colonization in GF mice enhanced the HPA axis response to stress ${ }^{20}$. Moreover, basal serum cortisol levels were decreased in $E$. coli LPS-treated mice in current studies, we speculated that the feedback regulation of HPA axis might be involved.

E. coli LPS in gut has an immune stimulation function, and can establish immune tolerance in infants and reduce the incidence of autoimmune diabetes ${ }^{57}$. GF mice without gut-derived LPS may lack this immune tolerance. Furthermore, different gut bacteria-derived LPS can affect each other's immune stimulating functions ${ }^{57}$, and we speculated that the "depression microbiota" recipient mice, which exhibited increased relative abundances of Actinobacteria and decreased Bacteroidetes in gut ${ }^{14}$ may also have abnormal immune tolerance. In addition, the abnormal immune system can lead to abnormal behaviors and elevated serum corticosterone baseline levels ${ }^{58}$. Hence, we speculated that gut microbiota may affect the HPA axis reactivity by regulating immune tolerance.

There are several limitations in our study. First, we did not conduct gene overexpression or knockdown tests, to further verify that key glucocorticoid receptor pathway gene influences behaviors, thus, we will further verify the present results in future studies. Second, we did not perform proteomics analysis to explore the exact protein changes caused by the upregulated and downregulated genes. We will further validate our results by combining proteomics and metabolomics. Third, oral LPS can induce abnormal behavior and gene expression in current study, but the mechanism is not clear. We speculated it may be associated with abnormal immune system. In next step, we will further explore the mechanism of oral LPS treatment affecting the mouse behavior and gene expression. Fourth, other pathways may also affect the glucocorticoid receptor pathway genes, so further studies are required to clarify whether other pathways are involved in the expression of glucocorticoid receptor pathway genes.

In summary, absence of gut microbiota induced antianxiety- and antidepressant-like phenotypes in GF mice, and $E$. coli LPS-treated mice exhibited antidepressant-like behavior, whereas the "depression microbiota" recipient mice showed anxiety- and depressive-like behaviors ${ }^{14}$, suggesting the gut microbiota affects depressive- and anxiety-like behaviors. Furthermore, glucocorticoid receptor pathway genes were upregulated in the hippocampus of mice with antianxiety- and/or antidepressantlike phenotypes. However, they were downregulated in mice with anxiety- and depressive-like phenotypes. These results indicated that the gut microbiota may lead to behavioral abnormalities in mice through the 
glucocorticoid receptor pathway. In present study, we provided new insight into the molecular mechanisms by which gut microbiota influence depressive-like behavior and proposed a new treatment target against depression.

\section{Acknowledgements}

This work was supported by the National Key R\&D program of China (Grant No. 2017YFA0505700), the National Key R\&D Program of China (Grant No. 2016YFC1307200), the National Natural Science Foundation of China (Grant No. 81701361) and the China Postdoctoral Science Foundation funded project (Grant No. 2017M612924). We thank Michal Bell, PhD, from Liwen Bianji, Edanz Editing China (www.liwenbianji.cn/ac), for editing the English text of a draft of this manuscript.

\section{Author details \\ 1 Institute of Neuroscience and the Collaborative Innovation Center for Brain Science, Chongqing Medical University, Chongqing 400016, China. \\ ${ }^{2}$ Chongqing Key Laboratory of Neurobiology, Chongqing 400016, China. ${ }^{3}$ Department of Neurology, Yongchuan Hospital, Chongqing Medical University, Chongqing 402160, China. ${ }^{4}$ Department of Laboratory Animal Science, College of Basic Medical Sciences, Third Military Medical University, Chongqing 400038, China. ${ }^{5}$ Department of Nephrology, the Second Affiliated Hospital of Chongqing Medical University, Chongqing 400010, China. ${ }^{6}$ Key Laboratory of Clinical Laboratory Diagnostics (Ministry of Education), Chongqing, China. ${ }^{7}$ Department of Neurology, the First Affiliated Hospital of Chongqing Medical University, Chongqing 400042, China. ${ }^{8}$ South Australian Health and Medical Research Institute, Mind and Brain Theme, and Flinders University, Adelaide, SA, Australia}

\section{Conflict of interest}

The authors declare no conflict of interest.

\section{Publisher's note}

Springer Nature remains neutral with regard to jurisdictional claims in published maps and institutional affiliations.

Supplementary Information accompanies this paper at (https://doi.org/ 10.1038/s41398-018-0240-5).

Received: 10 January 2018 Revised: 19 June 2018 Accepted: 14 July 2018 Published online: 07 September 2018

\section{References}

1. Qin, J. et al. A human gut microbial gene catalogue established by metagenomic sequencing. Nature 464, 59-65 (2010).

2. Eckburg, P. B. et al. Diversity of the human intestinal microbial flora. Science 308, 1635-1638 (2005).

3. Lozupone, C. A., Stombaugh, J. I., Gordon, J. I., Jansson, J. K. \& Knight, R. Diversity, stability and resilience of the human gut microbiota. Nature $\mathbf{4 8 9}$, 220-230 (2012)

4. Clemente, J. C., Ursell, L. K., Parfrey, L. W. \& Knight, R. The impact of the gut microbiota on human health: an integrative view. Cell 148, 1258-1270 (2012).

5. Bauer, K. C., Huus, K. E. \& Finlay, B. B. Microbes and the mind: emerging hallmarks of the gut microbiota-brain axis. Cell Microbiol. 18, 632-644 (2016).

6. Cryan, J. F. \& O'Mahony, S. M. The microbiome-gut-brain axis: from bowel to behavior. Neurogastroenterol. Motil. 23, 187-192 (2011).

7. Rhee, S. H., Pothoulakis, C. \& Mayer, E. A. Principles and clinical implications of the brain-gut-enteric microbiota axis. Nat. Rev. Gastroenterol. Hepatol. $\mathbf{6}$, 306-314 (2009).

8. Mayer, E. A. Gut feelings: the emerging biology of gut-brain communication. Nat. Rev. Neurosci. 12, 453-466 (2011).

9. Cryan, J. F. \& Dinan, T. G. Mind-altering microorganisms: the impact of the gut microbiota on brain and behaviour. Nat. Rev. Neurosci. 13, 701-712 (2012).

10. Collins, S. M., Surette, M. \& Bercik, P. The interplay between the intestinal microbiota and the brain. Nat. Rev. Microbiol. 10, 735-742 (2012).
11. Byrne, G. et al. Prevalence of anxiety and depression in patients with inflammatory bowel disease. Can. J. Gastroenterol. Hepatol. 2017, 6496727 (2017).

12. Panara, A. J. et al. The incidence and risk factors for developing depression after being diagnosed with inflammatory bowel disease: a cohort study. Aliment. Pharmacol. Ther. 39, 802-810 (2014).

13. Sherwin, E., Sandhu, K. V., Dinan, T. G. \& Cryan, J. F. May the force be with you: the light and dark sides of the microbiota-gut-brain axis in neuropsychiatry. CNS Drugs 30, 1019-1041 (2016).

14. Zheng, P. et al. Gut microbiome remodeling induces depressive-like behaviors through a pathway mediated by the host's metabolism. Mol. Psychiatry 21 786-796 (2016).

15. Huo, R. et al. Microbiota modulate anxiety-like behavior and endocrine abnormalities in hypothalamic-pituitary-adrenal axis. Front. Cell Infect. Microbiol. 7, 489 (2017).

16. Bo Li et al. Metabolite identification in fecal microbiota transplantation mouse livers and combined proteomics with chronic unpredictive mild stress mouse livers. Transl. Psychiatry 8, 34 (2017).

17. Chen, J. J. et al. Effects of gut microbiota on the microRNA and mRNA expression in the hippocampus of mice. Behav. Brain. Res. 322, 34-41 (2017).

18. Zeng, L. et al. Microbiota modulates behavior and protein Kinase $C$ mediated CAMP response element-binding protein signaling. Sci. Rep. 6, 29998 (2016).

19. Clarke, G. et al. The microbiome-gut-brain axis during early life regulates the hippocampal serotonergic system in a sex-dependent manner. Mol. Psychiatry 18, 666-673 (2013).

20. Sudo, N. et al. Postnatal microbial colonization programs the hypothalamicpituitary-adrenal system for stress response in mice. J. Physiol. 558, 263-275 (2004).

21. Neufeld, K. M., Kang, N., Bienenstock, J. \& Foster, J. A. Reduced anxiety-like behavior and central neurochemical change in germ-free mice. Neurogastroenterol. Motil. 23, 255-264 (2011). e119.

22. Crumeyrolle-Arias, M. et al. Absence of the gut microbiota enhances anxietylike behavior and neuroendocrine response to acute stress in rats. Psychoneuroendocrinology 42, 207-217 (2014).

23. Javad Barouei, M. M. \& Deborah, M. Hodgson. Effect of maternal probiotic intervention on HPA axis, immunity and gut microbiota in a rat model of irritable bowel syndrome. PLOS. ONE 10, 1371 (2012).

24. Nilsson, D. et al. Replication of genomewide associations with allergic sensitization and allergic rhinitis. Allergy 69, 1506-1514 (2014).

25. Storey, J. A direct approach to false discovery rates. J. R. Stat. Soc. Ser. B Stat. Methodol. 64, 479-98 (2002).

26. Chapman, J. R. \& Waldenstrom, J. With reference to reference genes: a systematic review of endogenous controls in gene expression studies. PLOS. ONE 10, e0141853 (2015).

27. Wos-Oxley, M. et al. Comparative evaluation of establishing a human gut microbial community within rodent models. Gut Microbes 3, 234-249 (2012).

28. Zhao, Y., Xie, W., Dai, J., Wang, Z. \& Huang, Y. The varying effects of short-term and long-term corticosterone injections on depression-like behavior in mice. Brain Res. 1261, 82-90 (2009).

29. Rao, R. P., Anilkumar, S., McEwen, B. S. \& Chattarii, S. Glucocorticoids protect against the delayed behavioral and cellular effects of acute stress on the amygdala. Biol. Psychiatry 72, 466-475 (2012).

30. Nicolas, C. S. et al. The role of JAK-STAT signaling within the CNS. JAKSTAT 2 e22925 (2013).

31. Sun, S. L., Li, T. J., Yang, P. Y., Qiu, Y. \& Rui, Y. C. Modulation of signal transducers and activators of transcription (STAT) factor pathways during focal cerebral ischaemia: a gene expression array study in rat hippocampus after middle cerebral artery occlusion. Clin. Exp. Pharmacol. Physiol. 34, 1097-1101 (2007).

32. Grimley PM, Dong F, Rui H. Stat5a and Stat5b: fraternal twins of signal transduction and transcriptional activation. Cytokine Growth Factor Rev. https:/ doi.org/10.1016/s1359-6101(99)00011-8 (1999).

33. Natarajan, C., Sriram, S., Muthian, G. \& Bright, J. J. Signaling through JAK2-STAT5 pathway is essential for IL-3-induced activation of microglia. Glia 45, 188-196 (2004).

34. Furigo, I. C., Ramos-Lobo, A. M., Frazao, R. \& Donato, J. Jr. Brain STAT5 signaling and behavioral control. Mol. Cell. Endocrinol. 438, 70-76 (2016).

35. Nyberg, F. \& Hallberg, M. Growth hormone and cognitive function. Nat. Rev. Endocrinol. 9, 357-365 (2013).

36. Adeva, M. M. et al. Brain edema in diseases of different etiology. Neurochem. Int. 61, 166-174 (2012). 
37. Qiu, B. et al. Overexpression of aquaporin1 aggravates hippocampal damage in mouse traumatic brain injury models. Mol. Med. Rep. 9, 916-922 (2014).

38. Ueda, Y. U. H. et al. Wernicke encephalopathy in a chronic peritoneal dialysis patient--correlation between diffusion MR and pathological findings. No To Hattatsu 39, 210-213 (2007).

39. Kim, J. E. et al. Differential expressions of aquaporin subtypes in astroglia in the hippocampus of chronic epileptic rats. Neuroscience 163, 781-789 (2009).

40. Tran, N. D. et al. Aquaporin-1-mediated cerebral edema following traumatic brain injury: effects of acidosis and corticosteroid administration. J. Neurosurg. 112, 1095-1104 (2010).

41. Hoshi A YT et al. Characteristics of aquaporin expression surrounding senile plaques and cerebral amyloid angiopathy in Alzheimer disease. J. Neuropathol. Exp. Neurol. 71:750-759 (2012).

42. Chen, W. et al. The lysosome-associated apoptosis-inducing protein containing the pleckstrin homology (PH) and FYV domains (LAPF), representative of a novel family of $\mathrm{PH}$ and FYE domain-containing proteins, induces caspaseindependent apoptosis via the lysosomal-mitochondrial pathway. J. Biol. Chem. 280, 40985-40995 (2005).

43. Flatmark T, Terland,O. Cytochrome b561 of the bovine adrenal chromaffin granules: a high potential b-type cytochrome. Biochem. Biophys. 253; 487-491 (1971).

44. Srivastava, M., Pollard, H. B. \& Fleming, P. J. Mouse cytochromeb561: cDNA cloning and expression in rat brain, mouse embryos, and human glioma cell lines. DNA Cell Biol. 17, 771-777 (1998). 10.1089.

45. Cenacchi, L. et al. Heterologous production and characterisation of two distinct dihaem-containing membrane integral cytochrome b(561) enzymes from Arabidopsis thaliana in Pichia pastoris and Escherichia coli cells. Biochim. Biophys. Acta 1818, 679-688 (2012)

46. Jepma, M. et al. Neurocognitive function in dopamine-beta-hydroxylase deficiency. Neuropsychopharmacology 36, 1608-1619 (2011).
47. Miecz, D. et al. Localization of organic cation/carnitine transporter (OCTN2) in cells forming the blood-brain barrier. J. Neurochem. 104, 113-123 (2008).

48. Tamai, I. Pharmacological and pathophysiological roles of carnitine/organic cation transporters (OCTNs: SLC22A4, SLC22A5 and Slc22a21). Biopharm. Drug. Dispos. 34, 29-44 (2013).

49. Sims B et al. Regulation of AMP deaminase by phosphoinositides. J. Biol. Chem. 1999; https://doi.org/10.1074/jbc.274.36.25701.

50. Wong, M. et al. AMPD3 is associated with the malignant characteristics of gastrointestinal stromal tumors. Oncol. Lett. 13, 1281-1287 (2017).

51. Du, J. et al. The role of nutrients in protecting mitochondrial function and neurotransmitter signaling: implications for the treatment of depression, PTSD, and suicidal behaviors. Crit. Rev. Food Sci. Nutr. 56, 2560-2578 (2016).

52. Zheng, P. et al. Plasma metabonomics as a novel diagnostic approach for major depressive disorder. J. Proteome Res. 11, 1741-1748 (2012).

53. Zheng $P$ et al. Identification and validation of urinary metabolite biomarkers for major depressive disorder. Mol Cell Proteomics 12, 207-214 (2013).

54. $\mathrm{Wu}, \mathrm{Y}$. et al. Metabolomic analysis reveals metabolic disturbances in the prefrontal cortex of the lipopolysaccharide-induced mouse model of depression. Behav. Brain. Res. 308, 115-127 (2016).

55. Velloso, L. A., Folli, F. \& Saad, M. J. TLR4 at the crossroads of nutrients, gut microbiota, and metabolic inflammation. Endocr. Rev. 36, 245-271 (2015).

56. Ait-Belgnaoui, A. et al. Prevention of gut leakiness by a probiotic treatment leads to attenuated HPA response to an acute psychological stress in rats. Psychoneuroendocrinology 37, 1885-1895 (2012).

57. Vatanen, T. et al. Variation in microbiome LPS immunogenicity contributes to autoimmunity in humans. Cell 165, 842-853 (2016).

58. Smith, C. J. et al. Probiotics normalize the gut-brain-microbiota axis in immunodeficient mice. Am. J. Physiol. Gastrointest. Liver Physiol. 307, G793-802 (2014). 